\title{
Combined Aortic and Liver Injury: Treatment Dilemma
}

\author{
Krunal Desai, Sandeep Jain, Anupama Shome, Kritya Dubey \\ Department of Emergency Medicine and Trauma, Max Superspeciality Hospital, Saket, New Delhi, India.
}

\section{Corresponding Author: \\ Dr. Krunal V Desai \\ Email: krunaldesai3991@gmail.com}

This is an Open Access article distributed under the terms of the Creative Commons Attribution License (creativecommons.org/ licenses/by/3.0).

Received : September 19, 2019

Accepted : November 2, 2019

Published : November 30, 2019

\begin{abstract}
Background: Blunt aortic arch injury is known but a rare presentation to Emergency Department following trauma. When associated with other visceral injuries, it has a high mortality rate. Case Report: We had female patient with aortic arch injury and liver injury following road traffic accident. In view of hemodynamic stability, patient was managed conservatively for first 48 hours followed by aortic cover stent. Patient was put on anti-coagulant after 3 days of intervention in view of liver injury to prevent stent thrombosis. Patient was discharged in stable condition without complication after 10 days hospital stay. Conclusion: Usually in stable patients, blunt aortic injuries are treated after stabilization of solid organ injuries which usually takes 7-10 days. Timing of treatment for blunt aortic injuries in such cases have not been clearly defined in literature. Our patient received an early stent graft 48 hours after injury. We are presenting case regarding this treatment dilemma.
\end{abstract}

Keywords: Aorta, Liver, Nonpenetrating Wounds, Stents, Thrombosis, Traffic Accidents.

\section{Introduction}

Blunt aortic arch injury is known but a rare presentation to Emergency Department (ED) following trauma. When associated with other visceral injuries, it has a high mortality rate [1]. Treatment in such cases can be a challenge when risk of bleeding and prevention of thrombosis needs to be critically balanced. We present a case of blunt aortic arch and liver injury.

\section{Case Report}

A 50-year-old female was brought to ED with complaint of abdominal pain 6 hours after road traffic accident. She was a rear seat passenger of a car which struck a stationary truck from behind. The patient was treated in ED as per ATLS protocol. Her airway was patent, breathing nonlaboured, RR 18/min, $\mathrm{SpO}_{2} 99 \%$ on room air, pulse rate of 100/min with BP 100/60 mmHg. Peripheral pulses were present with no external hemorrhage. GCS was 15/15. Patient was put on intravenous
(IV) fluids and IV analgesic. On secondary survey, abrasion on right upper abdomen was the only positive findings. Chest X-ray revealed a widened mediastinum [Fig.1] and fasting ultrasound showed free fluid in hepatorenal space. As patient was hemodynamically stable, contrast enhanced CT scan of chest and abdomen was done which showed intra-luminal filing defect in arch of aorta [Fig.2] and grade II liver injury [Fig.3]. Nonoperative management of liver trauma was selected and patient was shifted to intensive care unit for close monitoring. Covered stent graft was applied over aortic injury by Interventional Radiologist [Fig.4] 48 hours after injury. Usually, in postoperative period patient needs anticoagulants to prevent stent thrombosis but in view of liver injury anticoagulation could be detrimental. Therefore, patient was managed without anticoagulant for first 3 days with close monitoring and then started on low molecular weight heparin. Meanwhile risk of deep venous thrombosis was prevented with mechanical compression device. The patient was 


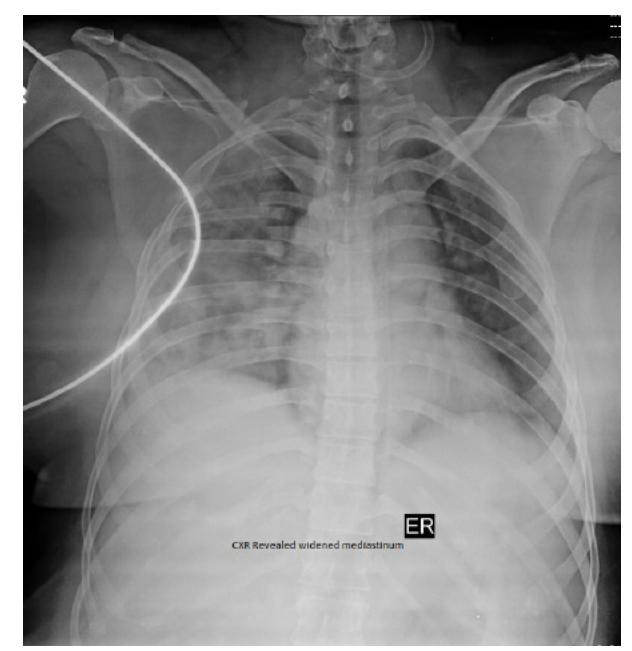

Fig.1: Widened mediastinum seen on chest X-ray.

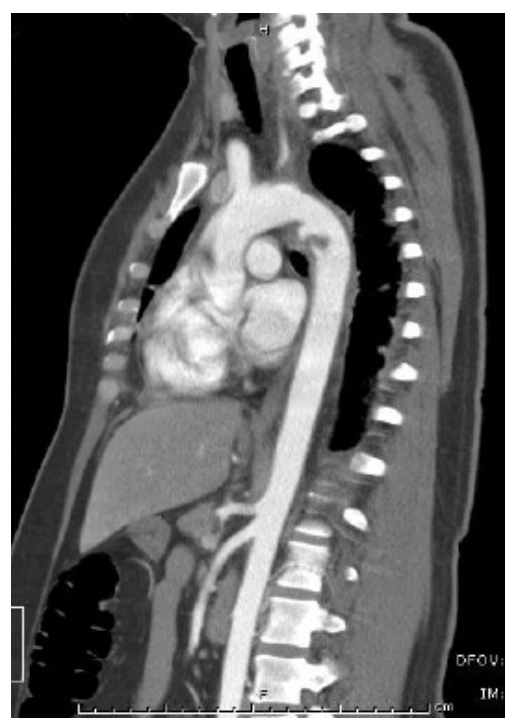

Fig.2: CECT chest shows intra-luminal filling defect with pseudoaneurysm in arch of aorta.

finally discharged in hemodynamically stable condition without any stent complications after 10 hospital days.

\section{Discussion}

Traumatic aortic arch injury is a life threatening injury which requires prompt diagnosis and treatment from Emergency Department. In 1958, Parmley looked at 296 cases of blunt aortic injury among young soldiers and found that those $15 \%$ that survive to get to hospital, $99 \%$ will die without

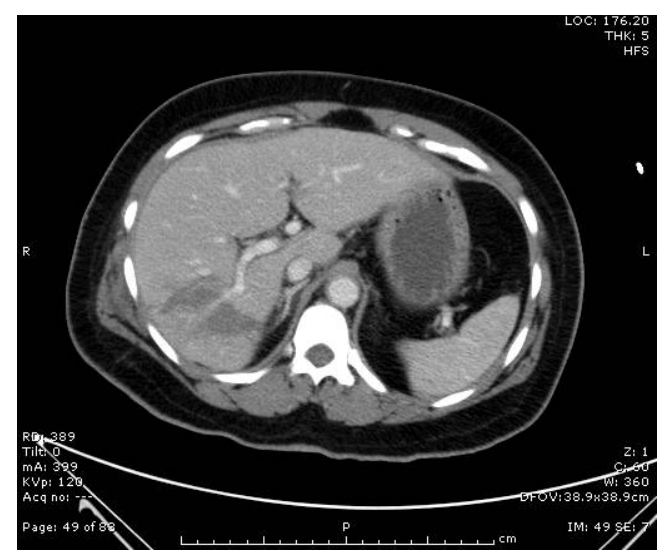

Fig.3: CECT abdomen shows grade II liver injury.

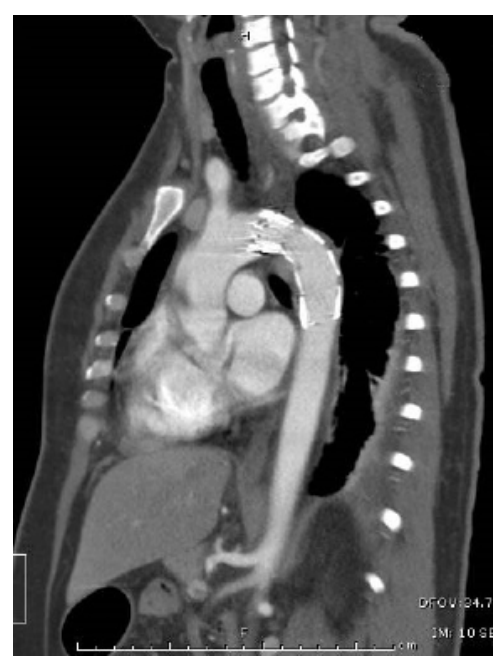

Fig.4: Covered stent applied over aorta.

surgical intervention. $15 \%$ survive only the first hour, 30\% die within six hours, 49\% die within 24 hours, $72 \%$ within eight days and $90 \%$ within four months [2]. These numbers revealed how fatal aortic injury is and require prompt diagnosis and treatment.

The proposed mechanisms for Traumatic Aortic Injury (TAI) are rapid deceleration, shearing forces, osseous pinch and hydrostatic forces or water-hammer phenomenon. Rapid deceleration in the lateral direction, antero-posterior direction, such as in head on collisions, causes shifting of the mediastinal structures sufficient to create shear stress on the aorta at the areas it is relatively 
immobile [3]. The osseous pinch mechanism is due to compression of the aorta between the spine and manubrium, first rib and/or medial clavicles [4]. The water-hammer effect is caused by a sudden increase in intra-thoracic pressure that can result in tears at the aortic isthmus or aortic root injury [5]. The most common associated injuries are multiple rib fractures which were seen in $75 \%$ of patients in one autopsy series. Sternal and first rib fractures are also fairly common in patients with TAI reflecting the significant force required to cause these injuries. Injuries to the heart, spleen and liver are also frequently seen in conjunction with aortic injury [6]. Assumption of aortic injury is always based on mechanism of injury. In our case, a possible deceleration injury due to vehicular collision was responsible

In most cases of TAI, clinical symptoms include chest pain, abdominal pain, dyspnea, cough, hoarseness of voice and dysphagia. Signs include anterior chest wall contusion, unexplained hypotension, upper limb hypertension or acute coarctation syndrome (decreased lower limb pulses with normal upper limb pulses), differences in pulse amplitude and a systolic murmur audible over the base of the heart or between the scapulae.

Chest x-ray is the first investigation of choice as a part of screening. Chest X-ray shows indirect signs like widened mediastinum (more than $8 \mathrm{~cm}$ when supine, or more than $6 \mathrm{~cm}$ when upright), indistinct or abnormal aortic contour, loss of the aorto-pulmonary window, widened paratracheal strip, large left hemithorax, deviation of trachea to right and depression of left main brainstem bronchus. CT chest aortogram has $100 \%$ sensitivity in confirming the diagnosis. It shows intra-luminal filling defect (intimal flap or clot), abnormal aortic contour (mural haematoma), pseudo-aneurysm or extravasation of contrast [8].

Traditionally open surgical repair was done but currently endovascular repair is the preferred modality of treatment. We also selected the same for our patient. A prospective multicenter study by the American Association for the Surgery of Trauma demonstrated lower mortality (7.2\% vs. $23.5 \%)$ and fewer blood transfusions in the endovascular repair group than in the open repair group. Other advantages of endovascular repair include lower risk of paraplegia and no need to open the chest cavity [8]. In minimal aortic injury, conservative management can be considered. It requires prompt attention, continuous hemodynamic monitoring, imaging study and blood pressure management.

In multiply injured patients, triaging of injuries for management is required. In hemodynamically stable patients, liver injuries are treated non-operatively. Highest risk of bleeding is in initial 72 hours. Blunt aortic injuries are treated after other life threatening injuries are stabilized, which may take upto 7-10 days [9]. Timing of treatment of blunt aortic injuries in such cases have not been clearly defined in literature. Our patient received an early stent graft 48 hours after injury. Anticoagulation is required in such cases to prevent stent thrombosis. We started low molecular weight heparin three days after stent placement to prevent bleeding from liver. Patient was discharged subsequently without any stent complications. Therefore, we believe that the timing of treatment and use of anticoagulants in such cases need to be investigated further.

\section{Conclusion}

Currently, non-operative management of liver trauma and endovascular repair of aortic arch injury is recommended treatment modality. Risk of bleeding from liver injury needs to be balanced with risk of stent thrombosis during initial period, for better patient outcome. Further studies are needed to define approach in these patients.

Contributors: KD: Manuscript writing. SJ: literature search and patient management; AS, KDu: manuscript editing and literature search. KD will act as a study guarantor. All authors approved the final version of this manuscript.

Funding: None; Competing interests: None stated. 


\section{References}

1. Arthurs ZM, Starnes BW, Sohn VY, Singh N, Martin MJ, Andersen CA. Functional and survival outcomes in traumatic blunt thoracic aortic injuries: an analysis of the National Trauma Databank. J Vasc Surg. 2009;49:988994.

2. Parmley LF, Mattingly TW, Manion WC. Nonpenetrating traumatic injury of the aorta. Circulation. 1953;17:10861101.

3. Feczko JD, Lynch L, Pless JE. An autopsy case review of 142 nonpenetrating (blunt) injuries of the aorta. J Trauma. 1992;33:846-849.

4. Cohen AM, Crass JR, Thomas HA. CT evidence for the "osseous pinch" mechanism of traumatic aortic injury. Am J Roentgenol. 1992;159:271-274.

5. Creasy JD, Chiles C, Routh WD. Overview of traumatic injury of the thoracic aorta. Radiographics. 1997;17:2745.

6. Williams JS, Graff JA, Uku JM. Aortic injury in vehicular trauma. Ann Thorac Surg. 1994;57:726-730.

7. O'Conor CE. Diagnosing traumatic rupture of the thoracic aorta in the emergency department. Emerg Med J. 2004;21(4):414-419.

8. Demetriades D, Velmahos GC, Scalea TM. Operative repair or endovascular stent graft in blunt traumatic thoracic aortic injuries: results of an American Association for the Surgery of Trauma Multicenter Study. J Trauma. 2008;64:561-570.

9. Tsukioka K, Kono K, Takahashi K, Kehara H, Urashita S, Komatsu K. A case of traumatic retrograde Type A aortic dissection accompanied by multiorgan injuries. Ann Vasc Dis. 2018;11(1):138-142. 